\title{
Rural Home-Stay Landscape Evaluation Based on the Method of AHP-Fuzzy Comprehensive Evaluation
}

\author{
Li Guangyao ${ }^{1,2}$ \\ ${ }^{1}$ College of Landscape Architecture, Nanjing Forestry University, Nanjing, China \\ ${ }^{2}$ College of Engineering, Zhejiang A \& F University, Hangzhou, China
}

\section{Email address:}

lgy@zafu.edu.cn

\author{
To cite this article: \\ Li Guangyao. Rural Home-Stay Landscape Evaluation Based on the Method of AHP-Fuzzy Comprehensive Evaluation. American Journal of \\ Civil Engineering. Vol. 4, No. 6, 2016, pp. 382-388. doi: 10.11648/j.ajce.20160406.27
}

Received: November 21, 2016; Accepted: December 22, 2016; Published: December 28, 2016

\begin{abstract}
The comprehensive evaluation is a significant part of rural home-stay landscape. Establishing a reasonable and accurate comprehensive system on rural home-stay landscape image, it will be benefit for making rural home-stay landscape characteristics and can promote the all-round development of them. On the basis of the current research results in the field of landscape evaluation, introducing the AHP-FCE evaluation theory and following the principle of choosing evaluation index, set up a comprehensive evaluation of rural home-stay landscape system. Using the comprehensive evaluation of ShangTianZhu home-stay landscape side of West Lake in Hangzhou China as a case and it prove the operability of the evaluation model.
\end{abstract}

Keywords: AHP, Fuzzy Comprehensive Evaluation, Rural Home-Stay, Home-Stay Landscape, Evaluation

\section{Introduction}

Based on agricultural resource, ecological environment and rural living culture, rural home-stay provides accommodation, rustic life show rural beautiful landscape and local customs and practices for tourists, offers some ways of experience in industrial economy, humanistic community and local's lifestyle. The characteristics of rural home-stay has two origins, the nature recover the original simplicity of living, the regional, local rural home-stay landscape image and cultural features. The nature of life do not has its own characteristics in every place, or even if it is difficult to express the most treasured meaning of local life, thus creating the source characteristics is mainly manifested in the landscape image of the environment resources, culture, custom, lifestyle, architecture feature etc. Currently, there is few study of rural home-stay landscape. According to R. Litton's study, rural home-stay landscape divides into back ground, middle ground and foreground in theory of the different viewer can enjoy different landscape of different relative distance. As the continuation of middle ground, back ground including the mountains, forest, waters, agricultural landscape and specific landform around the home-stay house, reveals relatively simple because of long distance. Middle ground including wooden fence, iron fence, hedge and wall is the most popular boundary material of business area, which shapes the form, color, type and surrounding. Foreground landscape being made of garden furniture, like grass, bonsai, pool and board-floor, it is innkeepers for outdoor leisure facilities for tourists, is the main part of the common landscape close to the hostel, meticulous landscape, shows its strong sense to visitors of color, material and design.

In the present, with the rapid development of rural homestay, there is an increasing required design of rural home-stay landscape. It helped local rural home-stay to improve the features, qualities and fame with excellent rural home-stay landscape planning and design. The accurate evaluation and judgment is an important part of rural home-stay landscape planning which helps to understanding the features and qualities in general. Assessment of rural home-stay landscape is a typical multi-factor integrative evaluation with part of fuzzy conclusion due to the balance between objectivity of feature and subjectivity of perception which influences factors. Therefore, in order to improve the assessment's reliability of the landscape esthetic evaluation, we need an idea to deal with multi-factor, fuzziness and subjective judgement.

We build up an assessment system called AHP-FCE model, using analytic hierarchy process and fuzzy comprehensive 
evaluation, to specifically analyze the weight of each feature factor account for, this system provide a method which combined with subjectivity, objectivity, qualitative analysis and quantification. The comprehensive assessment system contains four parts, in which every indicator is considered of nature of rural beauty and essential of feature creation. At last the system is tested with a real case and it get the result well agreed with field research and reality. To be exact, the assessment system is a success application.

\section{Methods}

This research mainly adopts the methods of documentary analysis, field investigation, questionnaire survey, and expert advice, the analytic hierarchy process, fuzzy comprehensive evaluation method, etc.

Questionnaire survey: collecting original data on landscape evaluation of rural home-stay feature.

AHP: used to determine the index of the previous level of weight.

FCE: evaluate the result of AHP comprehensively.

\subsection{The Analytic Hierarchy Process (AHP)}

After learning the ideas about assessing landscape, we use a modern scientific theory, analytic hierarchy process (AHP), to guide the assessment system, which is popularly used in assessment of resource evaluation or program. AHP is a qualitative and quantitative analysis phase combined of multi-target systematic and hierarchical analysis method. it provides an efficient and rigorous resolution, transforming qualitative problem into quantitative mathematical process, of complex and multi-factor decision, firstly analyzing complex fuzzy problem into some simple hierarchical structured factors, and then making decision from this factors. General application of AHP assessment system is:

Firstly, structure of value assessment system. We set up several layers in logic of target problem, named of target layer, standard layer, index layer and specific measures.

Secondly, judgement matrix constructing and consistence test. We calculate the total weight after every single weight of factor between two layers by matrix eigenvectors judgment. The plan, whose index owns max weight, is the best decision, as the more important index owns bigger weight. Process of rural home-stay landscape evaluation system building by using the AHP is actually the judgment for factors importance of creating rural home-stay landscape beauty. Now, according to the results of literature review and case analysis, construct the evaluation system of rural home-stay landscape should be catalogued target layer, criterion layer and index layer which by sorting the evaluation factors of the abstract degree and scope of the size, we can build the system structure (Figure 1) according to above theory. The system structure is a 3-layer assessment index system with first layer (Object), second layer (Criterion), third layer (Indicator).

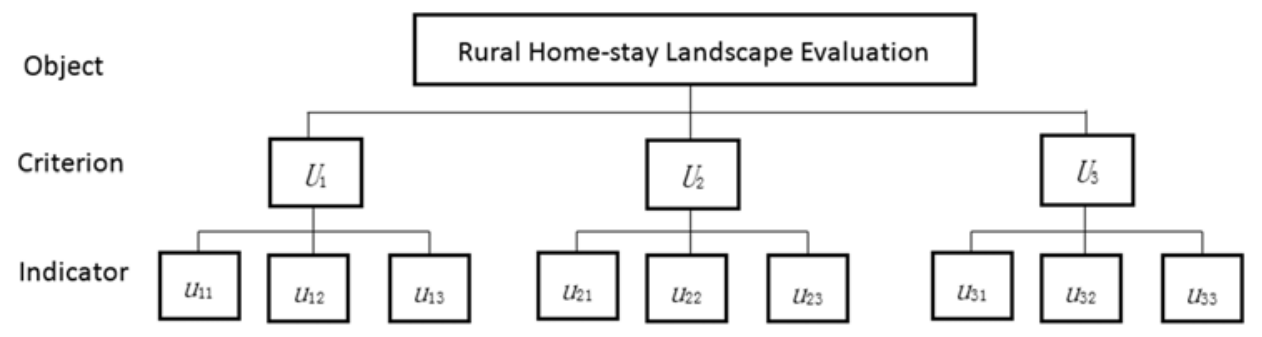

Figure 1. The structure of rural home stay landscape evaluation index system.

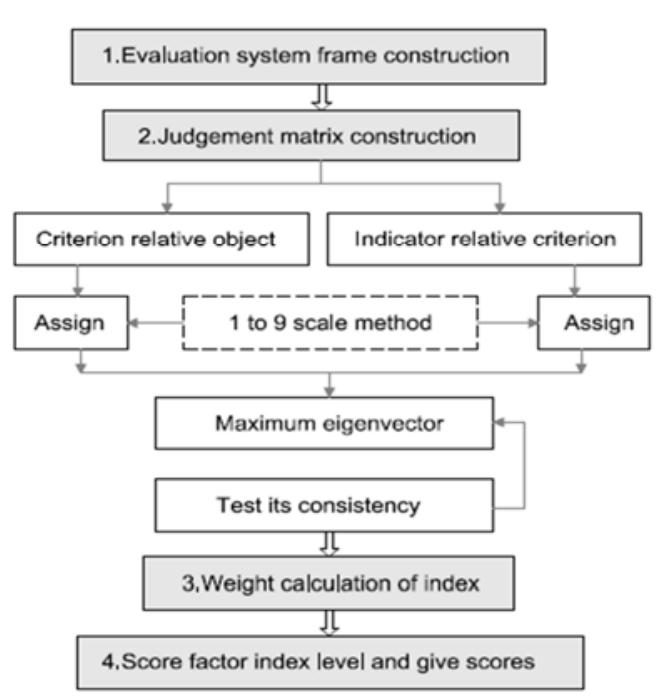

Figure 2. The step of rural home-stay landscape evaluation system construction with AHP.
Thirdly, weight calculation of every layers. We need to build judgment matrix between every two layers for quantifying importance of every layers' index, and calculate the every weight of it according to the calculation method of matrix.

Finally, set evaluation factors and weights. We set rating factors of index layer and bring it back to structure of assessment system.

Therefore, the construction can be divided into 4 steps: constructing the evaluation system framework, constructing judgment matrix and test its consistency, the weight calculation of every index, to score factor index level and given scores (Figure 2)

\subsection{Weight Vector Setting by AHP}

With analysis hierarchy process, planner would get weights of different factors from sample comparison and calculation among various factors on all layers. 


\subsubsection{Comparison Matrix Construction}

(1) Comparison scale construction

After determining the levels of factors, we need to judge the factor values by using pair-wise comparison. $\mathrm{u}_{\mathrm{ij}}$ used here to show the importance of lower factor $U_{i}$ to its upper factor $A_{k}$. In order to quantify the relative importance of assessment factors, we select the 1-9 scale of AHP and their reciprocals as scaled value. The meanings follow (table.1)

Table 1. Judgment matrix of 1-9 scale and meaning.

\begin{tabular}{|c|c|c|}
\hline Scale & Meaning & Explanation \\
\hline$u_{i j}=1$ & Indicate factor $\mathrm{Ui}$ is as important as factor $\mathrm{Uj}$ & \multirow{5}{*}{$\begin{array}{l}\text { The scaling values can be taken from the number } \\
2,4,6,8 \text {, and the mean value between the two } \\
\text { odd numbers. } \\
\text { The scale value can also take their reciprocal, if } \\
\text { the factor Ui and Uj compared to uij, then the } \\
\text { factor Ui and Uj compared to } 1 / \text { uij. }\end{array}$} \\
\hline$u_{i j}=3$ & Indicate factor $\mathrm{Ui}$ is slightly more important than factor $\mathrm{Uj}$ & \\
\hline$u_{i j}=5$ & Indicate that factor $\mathrm{Ui}$ is more important than factor $\mathrm{Uj}$ & \\
\hline$u_{i j}=7$ & Indicate that factor $\mathrm{Ui}$ is more important than factor $\mathrm{Uj}$ & \\
\hline$u_{i j}=9$ & Indicate factor $\mathrm{Ui}$ is extremely important than factor $\mathrm{Uj}$ & \\
\hline
\end{tabular}

(2) Judgement matrix construction

According to hierarchical model and relevant information, we construct judgement matrix $\mathrm{R}$ by experts' assessment of rural home-stay landscape features factors importance important degree evaluation by using table 1 .

$$
R=\left\{\begin{array}{ccc}
u_{11} & \cdots & u_{1 m} \\
\vdots & & \vdots \\
u_{n 1} & \cdots & u_{n m}
\end{array}\right\}
$$

\subsubsection{The Weight Vector Calculation}

In order to get the ordering weight of the relative factor on the layer which opposed to the factor on upper layer, we should rank the resulting matrix R. And then we calculate the maximum eigenvalue $\lambda \max$ and its corresponding eigenvector of matrix R. Common methods of single sequence are characteristic root, RMS, product legality. Because of the large amount of factors in discuss, we use a kind of simple method, RMS, to solve the normalized eigenvectors and eigenvalues of $\mathrm{R}$ until the consistency check. Calculated eigenvector is the ordering of every factors. The method fellows:

(1) Factors calculation of each row in judgement matrix.

$$
m_{i}=\prod_{j=1}^{n} a_{i j}(i=1,2, \cdots, n)
$$

(2) Calculate n-th root of $\omega_{i}$.

$$
\omega_{i}=\sqrt[n]{m_{i}}(i=1,2, \cdots, n)
$$

(3) Normalizing the eigenvector

$$
\begin{gathered}
\omega=\left(\omega_{1}, \omega_{2}, \cdots, \omega_{n}\right)^{T} . \\
\bar{\omega}=\frac{\omega_{i}}{\sum_{j=1}^{n} \omega_{j}}(i=1,2, \cdots, n)
\end{gathered}
$$

(4) The consistency check of matrix judgement.

Because of the complexity and uncertainty of the actual problems, which easily lead to wrong decisions when a large amount of factors are involved, we need to do consistency test of the judgement matrix to ensure actual result. The specific method is:

Firstly, calculating the max characteristic root

$$
\lambda_{\max }=\sum_{i=1}^{n}\left(R_{\omega}\right)_{i} / n \omega_{i}
$$

In this formula, $\left(\left(R_{\omega}\right)_{i}\right.$ means $\mathrm{i}$-th element of vector $\left.R_{\omega}\right)$. Secondly calculating the consistency proportion,

$$
\mathrm{CI}=\frac{\lambda_{\max }-n}{n-1}, \quad \mathrm{CR}=\mathrm{CI} / \mathrm{RI}
$$

In this formula, (CR is random consistency proportion; $\mathrm{CI}$ and RI is the consistency index and the average random consistency index). $\mathrm{CR}<0.1$, judgement matrix is in line with consistency. Otherwise, the matrix needs to be adjusted until the requirement is met (table 2).

Table 2. Values of average stochastic coincidence indicators.

\begin{tabular}{llllllllll}
\hline $\mathbf{N}$ & $\mathbf{1}$ & $\mathbf{2}$ & $\mathbf{3}$ & $\mathbf{4}$ & $\mathbf{5}$ & $\mathbf{6}$ & $\mathbf{7}$ & $\mathbf{8}$ & $\mathbf{9}$ \\
\hline $\mathrm{RI}$ & 0.00 & 0.00 & 0.58 & 0.90 & 1.12 & 1.24 & 1.32 & 1.41 & 1.45 \\
\hline
\end{tabular}

\section{Fuzzy Comprehensive Evaluation (FCE)}

Fuzzy comprehensive evaluation (FCE) is such thing that has comprehensively considered a variety of factors, or in another word, the system to make comprehensive and quantitative assessment. It has the characters of clean result and strong systematisms which works well on solving the problem of fuzzy and difficult to quantify, difficult of involved non-deterministic factors of rural home-stay landscape, accuracy improvement of assessment and decision.

\subsection{The Factor Set and Programme Set Establishment}

Assessment factor is a collection of all indexes which impact assessment target. Given that the number of impacted factors are $\mathrm{m}$, they constitute a set of factors is recorded as $U=\left\{U_{1}, U_{2}, \cdots U_{n}\right\}$; Setting assessment levels in number of $\mathrm{n}$ to make up a level set as $V=\left\{V_{1}, V_{2}, \cdots V_{n}\right\}$ is next. In the study, we set 5 levels for landscape aesthetic of rural home-stay, as follows: $V=\left\{V_{1}(\right.$ Best $), V_{2}($ Better $), V_{3}($ Good $), V_{4}($ Bad $), V_{5}($ Worse $\left.)\right\}$ 


\subsection{Subordinate Function Establishment}

Homogeneous evenly distributed subordinate sunction selected as following:

$$
\begin{gathered}
u_{i\left(x_{i}\right)}= \begin{cases}1, & X_{i}<x_{i 1} \\
0, & X_{i} \geq x_{i 1}\end{cases} \\
u_{j\left(x_{i}\right)}= \begin{cases}1, & X_{i} \leq x_{i 1}<x_{i, \mathrm{j}+1} \\
0, & X_{i}>x_{i j} \geq x_{i, \mathrm{j}+1}\end{cases} \\
u_{m\left(x_{i}\right)}= \begin{cases}1, & X_{i}<x_{i, m-1} \\
0, & X_{i} \geq x_{i, m-1}\end{cases}
\end{gathered}
$$

In the formula, $x_{i 1}, x_{i 2}, x_{i j}, x_{i, m-1}$ are grade standards of index in number $\mathrm{i}(\mathrm{i}=1,2, \ldots, \mathrm{m})$ on grade $\mathrm{j} . x_{i}$ is the basal data of index in the number $\mathrm{i}$-th; $u_{j\left(x_{i}\right)}$ is the membership degree of one indicator on grade $\mathrm{j}$.

\subsection{Single Factor Fuzzy Evaluation}

Single factor fuzzy evaluation is the evaluation to determine the factor membership to concentrated elements from a single factor. Given that evaluation object as i-th factor $U_{i}$, and membership as $C_{i j}$ of $\mathrm{j}$-th factor $\mathrm{v}_{\mathrm{j}}$, the result can be expressed as fuzzy sets: $R_{i}=\left\{r_{i 1}, r_{i 2}, \cdots, r_{i n}\right\}$ $i=(1,2, \cdots, n) . R_{i}$ is a single-factor evaluation set. Fuzzy matrix $\mathrm{R}$ is a one-factor evaluation matrix, composed of judgement set's membership of every factors, written following:

$$
R=\left[\begin{array}{cccc}
r_{11} & r_{12} & \cdots & r_{1 n} \\
r_{21} & r_{22} & \cdots & r_{2 n} \\
\cdots & \cdots & \cdots & \cdots \\
r_{m 1} & r_{m 2} & \cdots & r_{m n}
\end{array}\right]
$$

\subsection{First-Level Fuzzy Comprehensive Evaluation}

If the importance of every factor is same, which the concentrated weights are equal, it will get the factors' score of evaluation set by adding the factors of matrix R. Otherwise, it needs to do fuzzy matrix calculation and gets $\mathrm{B}$ as conclusion vector of first-level judgement standard:

$$
B=A \circ R
$$

In the formula, "॰" means some kind of synthetic arithmetic.

\subsection{Second-Level Fuzzy Comprehensive Evaluation}

As for the rural home-stay landscape assessment system is a multi-level structure, it needs to do multi-level fuzzy comprehensive evaluation. Multi-level comprehensive evaluation is that doing second-level comprehensive evaluation whose single factor evaluation matrix composed of the conclusion vectors of first-level comprehensive evaluation. Evaluation matrix of second-level comprehensive evaluation is as follows:

$$
R_{\text {Second }}=\left[\begin{array}{c}
B_{1 \text { First }} \\
B_{2 \text { First }} \\
\vdots \\
B_{m \text { First }}
\end{array}\right]=\left[\begin{array}{c}
A_{1} \circ R_{\text {lFirst }} \\
A_{2} \circ R_{2 \text { First }} \\
\vdots \\
A_{m} \circ R_{m \text { First }}
\end{array}\right]
$$

The conclusion vector of second-level judgement standard is:

$$
\begin{aligned}
& B_{\text {Second }}=A_{\text {Second }} \circ\left[\begin{array}{c}
A_{1} \circ R_{1 \text { First }} \\
A_{2} \circ R_{2 \text { First }} \\
\vdots \\
A_{m} \circ R_{\text {mirst }}
\end{array}\right] \\
& =\left[b_{1 \text { Second }}, b_{2 \text { Second }}, \cdots, b_{n \text { Second }}\right]
\end{aligned}
$$

In the calculation, $R_{\text {Second }}$ is the single factor judgement matrix of second-level comprehensive evaluation; $B_{i \mathrm{First}}$ is the matrix composed of the conclusions of first-level comprehensive evaluation; $A_{i}$ is the weight vector of firstlevel evaluation; $R_{i}$ is the first-level single factor judgement matrix; $B_{\text {Second }}$ is the conclusion vector of second-level comprehensive evaluation; $A_{\text {Second }}$ is the weight vector of second-level evaluation; $B_{j \text { Second }}$ is the grade evaluation result of rural home-stay landscape on the principle of maximum subordination whose assessment target is under the rule of second-level factors evaluation.

\section{Landscape Aesthetic Assessment for Rural Home-Stay}

\subsection{Principle of Assessment Index}

In order to build the assessment system which comprehensively reflects every sides of rural home-stay landscape's aesthetic sense, we should follow the general principle of systematic, scientific, dynamic, effectiveness, and the following three aspects:

Requirement of feature characters. Rural home-stay landscape has various types which are different from urban landscape or natural landscape. The characters are farmlands, orchards, natural scenery, characteristic houses, local custom and the sized and mixed units of houses and farmlands. Thus, we should select index of the rural home-stay landscape assessment which sufficiently takes account into characteristics and actual conditions.

General standards of beauty. The people who living in the city, looking forward to close and return to nature, so, the folk tourism, rural home-stay came into being. According to Marc Antrop's analysis, landscape aesthetic evaluation takes following parts: Firstly, the appropriate spatial scale; Secondly, Ordering of landscape structure, ordering is an expression of the relationship between landscape factors and human cognition, which moderate ordering, at small amount, 
is vivid and beneficial for landscape; Thirdly, landscape diversity, diversity of landscape types and dynamic change of space-time; Fourthly, sustainability and nature, the development and utilization of landscape thought should be reflected and its natural characteristics maintained.

System index should be comparability, quantifiability, operability. Comparability is the comparability of time and space. Comparability of time shows the development footprint of rural landscape; Comparability of space, shows the advantages and disadvantages between different rural landscape. Then, we can get appropriate conclusion and suggestion by index whose statistics, definitions and scope remain same in different time and different place. Quantification is required to quantify the qualitative indicators can be indirectly assigned. Operability, it includes method, information and index, in which choice of statistical method and analysis of mathematical model could be easy to build.

\subsection{General Structure of Index System}

According to analytic hierarchy process (AHP) and fuzzy comprehensive evaluation theory (FCE) in the analysis of complex problems, we should stratify factors included in the issue as the first step in system's characteristics, which summarize involved factors and their relations by a hierarchical model. Thus, we build a 3-layer assessment index system of rural home-stay landscape based on the principle of index selecting and general judgement of rural home-stay landscape aesthetics, with first layer (Object), second layer (Criterion), third layer (Indicator). The first layer has only one factor that analyzing the intended goal or ideal results; the second layer is the middle step for achieving goals which composed of layers of criteria and sub-criteria; the third layer consists of a variety of measures and decision programs. In the study, assessment system is made of 3 layers, first layer (Object) of comprehensive assessment index of rural home-stay landscape, second layer (Criterion) of four functions' index in rural home-stay facilities landscape, rural home-stay ecological landscape, rural home-stay humanistic landscape and rural home-stay aesthetic conception, third layer (Indicator) of selected 12 specific index. (table 3 )

Table 3. Evaluation index system about the home-stay landscape.

\begin{tabular}{|c|c|c|c|c|}
\hline \multirow{2}{*}{ Object } & \multirow{2}{*}{ Criterion } & \multirow{2}{*}{ Indicator } & \multicolumn{2}{|c|}{ Indicator weight } \\
\hline & & & Single sort & Whole sort \\
\hline \multirow{12}{*}{$\begin{array}{l}\text { comprehensive } \\
\text { assessment } \\
\text { index of rural } \\
\text { home-stay } \\
\text { landscape A } \\
(1.0)\end{array}$} & \multirow{4}{*}{$\begin{array}{l}\text { rural home-stay facilities } \\
\text { landscape B1 }(0.3119)\end{array}$} & design artistry of landscape facilities (C11) & 0.5246 & 0.1636 \\
\hline & & visual harmony of landscape facilities (C12) & 0.2052 & 0.0640 \\
\hline & & coordination degree of landscape facilities and the surrounding (C13) & 0.1824 & 0.0569 \\
\hline & & landscape facility integrity and continuity (C14) & 0.0879 & 0.0274 \\
\hline & \multirow{5}{*}{$\begin{array}{l}\text { rural home-stay ecological } \\
\text { landscape B2 }(0.3373)\end{array}$} & landscape level richness (C21) & 0.4523 & 0.1526 \\
\hline & & plant seasonal and color aspect (C22) & 0.0834 & 0.0282 \\
\hline & & landscape harmony and artistic composition (C23) & 0.3298 & 0.1078 \\
\hline & & regional feature of geological sights $(\mathrm{C} 24)$ & 0.1445 & 0.0487 \\
\hline & & traditional folk customs heterogeneity (C31) & 0.1775 & 0.0381 \\
\hline & \multirow{2}{*}{$\begin{array}{l}\text { rural home-stay humanistic } \\
\text { landscape B3 }(0.2147)\end{array}$} & local culture artistry (C32) & 0.4336 & 0.0931 \\
\hline & & rural life culture experience (C33) & 0.3889 & 0.0835 \\
\hline & $\begin{array}{l}\text { rural home-stay aesthetic } \\
\text { conception B4 }(0.1361)\end{array}$ & embody the idea, enhance the state (C41) & 1.0000 & 0.1361 \\
\hline
\end{tabular}

\subsection{Comprehensive Assessments Factor System Construction}

On the assessment of landscape aesthetic, we need to obey the following principles: objectivity, subjectivity, comprehensiveness and awareness. The assessment of landscape quality would be influenced by the different feelings and different judgements of different people who has different character and aesthetic favor. Therefore, only if the influential factors, landscape quality, attraction, awareness, coordination, be accepted, we can evaluate the aesthetic assessment of most people. The aesthetic principles of rural home-stay landscape are following 6 parts: I. Plants and waters, which lead citizens to rural landscape with feeling of nature; II. Curiosity; III. Environment, or another name of ecological aesthetics; IV. Ordering, which demand the landscape space looked in the rule of perspective, coordination with background, concentration distribution and concealment; V. Diversity, people like various features; VI. Migration, including visitors' accessibility and freedom of bio-migration.

\section{Case Analysis}

\subsection{Introduction of Subject}

West Lake Reclusive Life hotel located on the edge of West Lake, southeast of temple Shangtianzhu Faxi, composed by three neighboring cottages, quiet and convenient, has several part of landscape space in the south garden which shaped by platforms, with the background, 
temples landscape. There are some characteristics in the landscape project:

(1) Creating a mood like home by retaining the primary track of daily life and designing of no wall for public connivance.

(2) Creating a combined traditional hermit culture of artistic realm and living environment. Axial space are designed in line of starting space (water feature), turning space (public garden) and ending space (meditation platform). In plant configuration, bamboo planting in the edge space is dense but the density properly, look from inside to outside, swaying ventilation thoroughly, the garden is small but it is one with the vision of the forest, intoxicated in this natural scenery.

(3) The building renovation design, facade, color, material, size, are follow unity with surroundings. The natural sound is introduced into the landscape space; slowly the water sound and evening drum and morning bell from the monastery enhance the appeal of the place. The smells of flower, shadows of trees and cloud decorate the space into harmony; therefor form a multidimensional experience of perception.

\subsection{Project Evaluation}

1) We invited 5 experts to judge the qualitative landscape index of West Lake Reclusive Life hotel based on assessment system. Following the weight set of evaluation index:

$\omega=(0.393,0.234,0.234,0.139), \omega_{U_{1}}=(0.243,0.243$, $0.342,0.172), \omega_{U_{2}}=(0.521,0.126,0.162,0.191), \omega_{U_{3}}=$ $(0.333,0.333,0.333), \omega_{U_{41}}=1.000$ 。

2) Fuzzy comprehensive evaluation (FCE) matrix based on the weight set is as follows:

$$
\begin{aligned}
& R_{1}=\left[\begin{array}{lllll}
1 / 5 & 4 / 5 & 0 & 0 & 0 \\
1 / 5 & 3 / 5 & 0 & 0 & 0 \\
1 / 5 & 2 / 5 & 2 / 5 & 0 & 0 \\
0 & 3 / 5 & 2 / 5 & 0 & 0
\end{array}\right], R_{2}=\left[\begin{array}{ccccc}
1 / 5 & 3 / 5 & 1 / 5 & 0 & 0 \\
0 & 2 / 5 & 3 / 5 & 0 & 0 \\
1 & 0 & 0 & 0 & 0 \\
0 & 1 & 0 & 0 & 0
\end{array}\right] \\
& R_{3}=\left[\begin{array}{ccccc}
0 & 2 / 5 & 0 & 0 & 0 \\
1 / 5 & 1 / 5 & 3 / 5 & 0 & 0 \\
1 / 5 & 2 / 5 & 2 / 5 & 0 & 0
\end{array}\right], R_{1}=\left[\begin{array}{lllll}
1 / 5 & 2 / 5 & 2 / 5 & 0 & 0
\end{array}\right]
\end{aligned}
$$

3) First level comprehensive evaluation turns to the first level comprehensive evaluation of first level index level, Ak:

$$
\begin{aligned}
& \mathrm{A}_{1}=\omega_{U_{1}} \times R_{1}=(0.166,0.580,0.206,0,0), \\
& \mathrm{A}_{2}=\omega_{U_{2}} \times R_{2}=(0.266,0.554,0.180,0,0), \\
& \mathrm{A}_{3}=\omega_{U_{3}} \times R_{3}=(0.133,0.333,0.533,0,0), \\
& \mathrm{A}_{4}=\omega_{U_{4}} \times R_{4}=(0.200,0.400,0.400,0,0) .
\end{aligned}
$$

4) Second level comprehensive evaluation turns to second level comprehensive evaluation of target level index level, A:

$$
\begin{aligned}
\mathrm{A}= & \omega \times R=\omega \times\left(\mathrm{A}_{1}, \mathrm{~A}_{2}, \mathrm{~A}_{3}, \mathrm{~A}_{4}\right)^{T} \\
& =(0.186,0.304,0.492,0,0) .
\end{aligned}
$$

5) Finally, we classified the landscape assessment result according to the principle maximum subordination. General result of the case is relatively beautiful. The individual comments from the A1and A3 reveal that, index of facilities landscape and cultural landscape scores high, in which facilities landscape got relatively beautiful, and cultural landscape scores between relatively beautiful and common beautiful. Assessment result fits to field observations.

\section{Conclusions and Recommendations}

1) On account of the assessment system having not been tested in other region of different aesthetic, we cannot say the system would perform well in every region. On the premise of rule unchanged, we suggest to reset the weight of factors based on specific condition. e.g., But all of the new index should be ensured that can reflect the rural landscape resources and local characteristics.

2) It is first time to use the analytic hierarchy process (AHP) and fuzzy comprehensive evaluation (FCE) at assessment of rural home-stay feature. The method is simple. The results, final outcome and outcome of index in every layer, are clear, not only can know the overall results of the completion of the landscape, but also to know the results of the evaluation of indicators at all levels. The assessment system benefit to both differing advantage from disadvantage and giving targeted proposals. The model, logical, systematic, practical, achieves the quantifiable study of nonquantitative factors, is a new solution can be applied to assessment of rural home-stay, and can be applied to other assessment of landscape aesthetic too.

3) With the development of rural tourism, rural home-stay feature construction has become an inevitable tendency. AHP and FCE deliver qualitative issues to quantitative method through a calculation rule. Some time, the data is part of subjective, size and background of specialists, drives to inaccurate outcome, which need to be improved.

\section{References}

[1] XU X Z. XUE J H. Aesthetic evaluation for plant landscape of wetland park based on AHP [J]. Journal of Northwest Forestry University. 2008. 27 (2): 213-216. (in Chinese).

[2] Li K I. Using analytic hierarchy process in urban road landscape evaluation [J]. Engineering Journal of Wuhan University. 2005 (1); 143-152. (In Chinese).

[3] Marc Antrop. Background concepts for integrated landscape. Analysis [J] Agriculture, Ecosystem and Environment. 2000: $77,17-18$. 
[4] Ismail MN, Hanafiah MH, Aminuddin N, Mustafa N. Community-based Homestay Service Quality, Visitor Satisfaction, and Behavioral Intention. Procedia-Social and Behavioral Sciences. 2016 Jun 23; 222: 398-405.

[5] Ogucha EB, Riungu GK, Kiama FK, Mukolwe E. The influence of homestay facilities on tourist satisfaction in the Lake Victoria Kenya Tourism Circuit. Journal of Ecotourism. 2015 Sep 2; 14 (2-3): 278-87.

[6] Shen, Ching-Cheng, and Der-Jen Liu. "Correlation between the homestay experience and brand equity." Journal of Hospitality and Tourism Technology 6.1 (2015): 59.

[7] ZHANG KX, DONG L. The Study on the Suitability Evaluation System of Extending the Opening Period of Urban Park_ A Case Study of Shanghai Urban Parks. Chinese Landscape Architecture. 2016; 7: 012.

[8] Ling YL, Zhu JD. Research about the Evaluation Index of Agricultural Sightseeing Garden Landscape Resources based on AHP. South Korean environmental science. 2016; 25 (4): 483-90.

[9] LUBETKIN M. Bed-and-breakfasts: Advertising and promotion [J]. Cornell Hotel and restaurant Administration Quarterly, 1999, 40 (4): 84-90.

[10] SAATY T L. The Analytic Hierarchy Process [M] New York: Megraw-Hill, 1980.

[11] ZOU Z H, YUN Y, SUN J N. Entropy method for determination of weight of evaluating indicators in fuzzy synthetic evaluation for water quality assessment $[\mathrm{J}]$. Journal of Environmental Sciences, 2006, 18 (5):1 020-1 023.
[12] Beerli A, Martin J D. Factors influencing destination image [J] Annals of Tourism Research, 2004, 31 (3): 657- 681.

[13] Gallarza M G, Saura I G, Garcia H C. Destination image: Towarda conceptual framework [J]. Annals of Tourism Research, 2002, 29 (1): 56-78.

[14] Chen JF, Hsieh HN, Do QH. Evaluating teaching performance based on fuzzy AHP and comprehensive evaluation approach. Applied Soft Computing. 2015 Mar 31; 28: 100-8.

[15] Li H, Qiang M, Yu-mei LU, Min J. Analysis and study on AHP-fuzzy comprehensive evaluation. China Safety Science Journal. 2004; 14 (7): 86-9.

[16] Li Y, Gong G, Li N. A comprehensive performance evaluation framework of complex products based on a fuzzy AHP and DS theory. International Journal of Modeling, Simulation, and Scientific Computing. 2016 Mar 29: 1650020.

[17] Aydogan EK, Demirtas O, Dagdeviren M. A New Integrated Fuzzy Multi-Criteria Decision Model for Performance Evaluation. Business and Management Studies. 2015 Feb 28; 1 (1):38-55.

[18] Wang, Ya-Jun, and Shan-Shan Yu. "Model for evaluating the rural landscape design schemes with fuzzy number intuitionistic fuzzy information." Journal of Intelligent \& Fuzzy Systems Preprint (2016): 1-10. 